\title{
Facile Synthesis and Characterization of Chitosan Nanofibers by Oil/Water Emulsion Method
}

\author{
D. Ragupathy ${ }^{* 1}$, E. Muthusankar ${ }^{1,2}$, D. Kamalakannan ${ }^{3}$ \\ ${ }^{1}$ Department of Nano Electro-Chemistry Lab (NEL), National Institute of Technology Puducherry, \\ Karaikal 609-609, India \\ ${ }^{1,2}$ Department of Electronics and Communication Engineering, National Institute of Technology Puducherry, \\ Karaikal 609-609, India \\ ${ }^{3}$ Department of Mechanical Engineering, Pondicherry Engineering College, Puducherry - 605014 , India \\ *Corresponding author email: ragu.nitpy@gmail.com; ragu@nitpy.ac.in
}

Received: 27 March 2018 / Accepted: 9 April 2018 / Published: 22 April 2018

\begin{abstract}
A facial approach for the synthesis of surfactant free, biodegradable and eco-friendly chitosan nanofibers (CS-NFS) was prepared by Oil/Water Emulsion method. The morphology of the CS-NFS was examined by field emission scanning electron microscopy (FE-SEM). FE-SEM images show the nanoscale chitosan nanofibers formation with sizes in the ranges of $\sim 100-200 \mathrm{~nm}$. Physiochemical characterizations of the CS-NFS were analyzed by Fourier transform infrared spectroscopy (FT-IR), UV-visible spectroscopy and thermogravimetric analysis. The CS-NFS are expected to be useful in electrical, optical and electrochemical devices.
\end{abstract}

Keywords: Chitosan. Surfactant free. Electrical. Nanofibers

\section{Introduction}

In research and scientific upgrading developments nanotechnology plays a historic task for the past numerous decades. Nanomaterials are materials with essential structural units, grains, particles, fibers or other constituent components smaller than $100 \mathrm{~nm}$ in at least one dimension and these nanomaterials can be made of metals, ceramics, polymers, organic materials and composite thereof, just like predictable or micron structured materials. Nanomaterials consist of nanoparticles, nano clusters, nanocrystals, nanotubes, Nanofibers, nanowires, nanorods, nanofilms, etc. So far abundant bottom-up and top-down nanofabrication technologies (such as electro spinning, phase separation, self-assembly process, chemical vapor deposition and chemical etching) are accessible to generate nanomaterials with controlled or random nanotopographies [1].

In topical material research studies, nanofiber technology was most important and precious object in the modern investigation [2]. Among the Nanomaterials, nanofibers have amazing characteristics such as exceptionally minute pore size with very outsized surface area-to-volume proportion, high porosity and diameters of the fiber was in nanometer sequence. So nanofibers can be capable materials for numerous biomedical and potential applications such as tissue templates, medical prostheses, artificial organ, wound dressings, drug delivery, pharmaceutical composition and filters, protective clothing, reinforcement in composite materials and sensor [3,4]. Owing to the current existence, there has been an increasing curiosity in edible and biodegradable fibers based on biopolymers. In these edible biopolymers, polysaccharide based multifunctional biomaterials find a continuous and growing interest. Among polysaccharides, glycosaminoglycans (chitosan) are particularly interesting for their bioactive properties $[5,6]$. The linear amino polysaccharide chitosan, are particularly attractive not only as abundant biomass resources but also as specialty biopolymers for preparing advanced functional materials. 
Facile Synthesis and Characterization of Chitosan Nanofibers by Oil/Water Emulsion Method

Chitosan (CS) is the product of the partial deacetylation of the naturally occurring marine versatile biopolymer-chitin, which is found in the exoskeletons of insects and marine invertebrates [7]. Chitosan is composed primarily of glucosamine, 2-amino-2-deoxy-b-D-glucose, known as (1/4)-2-amino-2-deoxy-(Dglucose) [8-11]. The positive attributes of chitosan have admirable biocompatibility and excellent biodegradability with ecological safety and non-toxicity with versatile biological activities such as antimicrobial activity and low immunogenicity have provided generous opportunities for advance development. It has become of huge attention not only as an under-utilized resource but also as an efficient biomaterial of high potential in various fields, for example, biotechnology, wastewater treatment, cosmetics and biomedical areas as drug delivery vehicles, carriers of immobilized enzymes and cells, biosensors, artificial organs, orthopedic materials, surgical devices, biodegradable packaging, and in particular, temporary implants for fixations and supports in tissue regeneration [12-14]. Consequently, CS based nanomaterials finds various application and development for the future.

There have been a number of earlier attempts at reviewing the region on chitosan fibers covering certain aspects of their importance, properties and applications. Chitosan fibers situate apart from all other biodegradable natural fibers in several inherent properties such as biocompatibility, non-toxicity, biodegradability, low immunogenic, non-toxicity, etc. These properties in combination with good mechanical properties make them good candidate materials for sutures that form the largest groups of material implants used in human body [15-17]. CS fibers have been found to be valuable useful in other medical textiles [18], wound dressing [19] and haemostatic materials [20] and several other prosthetic devices such as aemostatic clips, vascular and joint prostheses, mesh and knit abdominal thoracic wall replacements and as antimicrobial agents [21].

Earlier, the nanofibers were produced by several fabrication techniques, like electro spinning [22], meltblown [23], phase separation [24], self-assembly [25] and template synthesis [26]. Compared to other methods, interfacial polymerization is a powerful, inexpensive and simple method for the preparation of polymer based nanofibers [27-29].

The present work investigates the potential use of CS nanofiberous scaffolds synthesize by simple Oil/Water Emulsion method. The low and high MW of chitosan nanofibrous matrix with different ratios was synthesized for comparative purposes. The morphology of the nanofibers was analyzed with an average diameter about $\sim 100-200 \mathrm{~nm}$. The structural characterization was analyzed using FTIR and UV-Visible spectroscopy. Themogravimetic analysis was exploited to determine the thermal property of the fibers. To the best of our knowledge, reports on the synthesis of CS nanofibers by Oil/Water Emulsion method are scarce.

\section{Materials and Methods}

\subsection{Material}

Chitosan (low molecular weight (LMW); $\left.\mathrm{C}_{6} \mathrm{H}_{11} \mathrm{NO}_{4}\right)_{\mathrm{n}}$ \& (high molecular weight $\left.\mathrm{HMW} ; \mathrm{C}_{12} \mathrm{H}_{24} \mathrm{NO}_{4}\right)_{\mathrm{n}}$ were obtained from Wako. Petroleum ether (PE) was used as an organic solvent. Distilled water was used as aqueous phase. Ammonium persulphate (APS), hydrochloric acid and all other reagents were analytical grade and used without further purification.

\subsection{Synthesis of Chitosan Nano Fibrous Scaffolds (CS-NFS)}

Chitosan nano fibrous scaffolds (CS-NFS) were prepared by in the interface of two immiscible solvents (water/petroleum ether). The by-products of the result are easily separated owing to their solubility in the aqueous or the organic solvents. The condensed hydrocholoric acid $(\mathrm{HCl}, 3.0 \mathrm{~mL})$ and ammonium persulphate (APS, $1.14 \mathrm{~g}$ ) were dissolved in $100 \mathrm{~mL}$ of distilled water in a $500 \mathrm{~mL}$ beaker, and then petroleum ether $(\mathrm{PE}, 100 \mathrm{~mL})$ was added to the surface of the water solution. After ten minutes later, LMW chitosan $(20,40,60,80 \mathrm{mM})$ mixed with $2 \%$ aqueous acetic acid was added carefully to the petroleum ether and then reaction was started at aqueous/organic interface of water and petroleum ether. The reaction was conceded out under room temperature for 3 days without stirring. The precipitate was centrifuged and 
Dhanusuraman et al., Adv. Nan. Res.; Vol. 1, Issue 1, pp: 31-37, 2018

washed with distilled water for several times to remove all other impurities. The consequential products were dried at room temperature for 24 hours. Thus, CS-NFS was synthesized. The procedure as mentioned above was adopted to prepare high MW chitosan NFS.

\subsection{Instrumentation}

The morphology of CS-NFS was investigated using field emission scanning electron microscopy (FE-SEM) (TEOL JSM-5600LV instrument with the operation of $25 \mathrm{kV}$ ). Fourier transform infrared spectrometer (FTIR) (Nicolet, USA) was used to record the spectra of CS-NFS using KBr pellets. Thermal stability (TGDTG) (Perkin Elmer) of samples was made at a heating rate of $10^{\circ} \mathrm{C} / \mathrm{min}$ under a nitrogen atmosphere over a temperature range of $30-800{ }^{\circ} \mathrm{C}$. Shimadzu UV-1700 model UV-vis spectrophotometer was evaluated to analyze the fibers.

\section{Results and Discussion}

\subsection{Morphology}

The FE-SEM images show the morphology of CS-NFS prepared with different CS concentrations. Fig.1 shows the FESEM images of low molecular weight CS-NFS at different concentration from 10 to $40 \mathrm{mM}$ (Fig.1a-d). We could find a little fiber in fig.1a. Fig.1b shows the fine and uniform formation of CS-NF diameter in the range of $100-200 \mathrm{~nm}$. Fig1.c and d show the aggregation of nanofibers to form bundled shaped fibers. While increasing the molar ratios of the chiotsan monomer the aggregation of the fibers tends to form bundles. As compared to other molar ratios of CS (10, 30 and 40 mM; Fig.1a, c and d), 20 $\mathrm{mM}$ shows the well orderly arranged nanofibers were found. While decreasing the lower concentration of chitosan to below $10 \mathrm{mM}$, we could find a film like morphology. Thus, from the FESEM image it is concluded that $20 \mathrm{mM}$ is an excellent molar ratio for the CS-NFS formation.
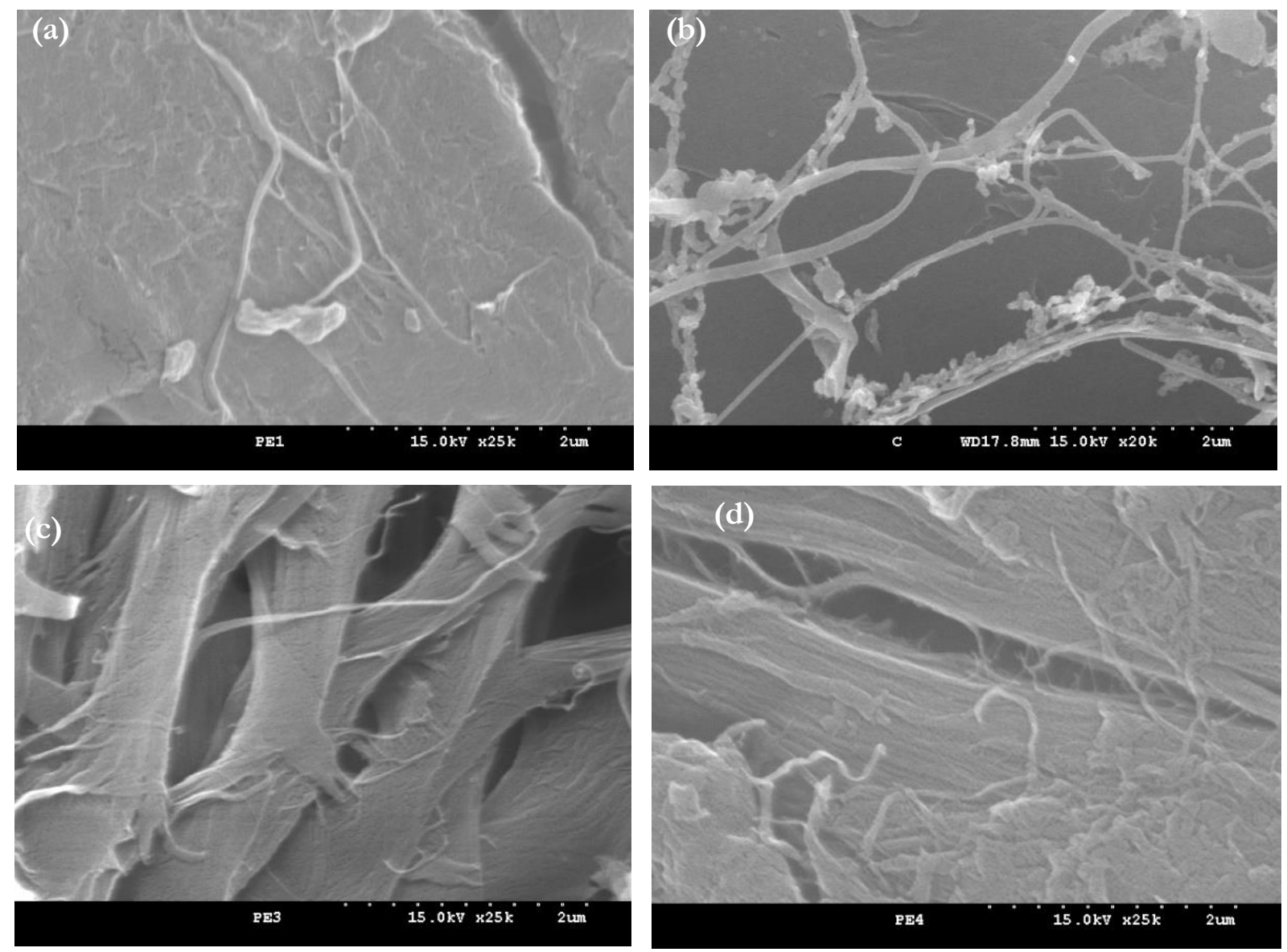

Figure1: FESEM images of CS-NFS/LMW at different molar ratios (a) 10 (b) 20 (c) 30 and (d) 40 
Fig. 2 presents the FESEM images CS-NFS/HMW at different concentration from 10 to $40 \mathrm{mM}$ (Fig.1a d). Relatively homogeneous, continuous and randomly oriented fiber marix were obtained under the optimized conditions for $10 \mathrm{mM}$ (Fig. 2a), $20 \mathrm{mM}$ (Fig. 2b), $30 \mathrm{mM}$ (Fig. 2c) and $40 \mathrm{mM}$ (Fig. 2d). As the solution concentration increased, the average fiber diameter linearly increased for all of the HMW chitosan samples. The diameter of the fiber was interrelated with chitosan ratios and solution concentrations. The ranges of the diameter distributions become fine for the HMW samples. While decreasing the lower concentration of chitosan to below $10 \mathrm{mM}$, higher quantity of fiber obtained compared to lower MW chitosan. These results indicate that the HMW chitosan were successfully fabricated into thinner and homogeneous fibers compared to those obtained using lower molecular weight chitosan. The ability of the fiber forming property was superior for the HMW chitosan than the lower molecular weight chitosan at very low concentrations.
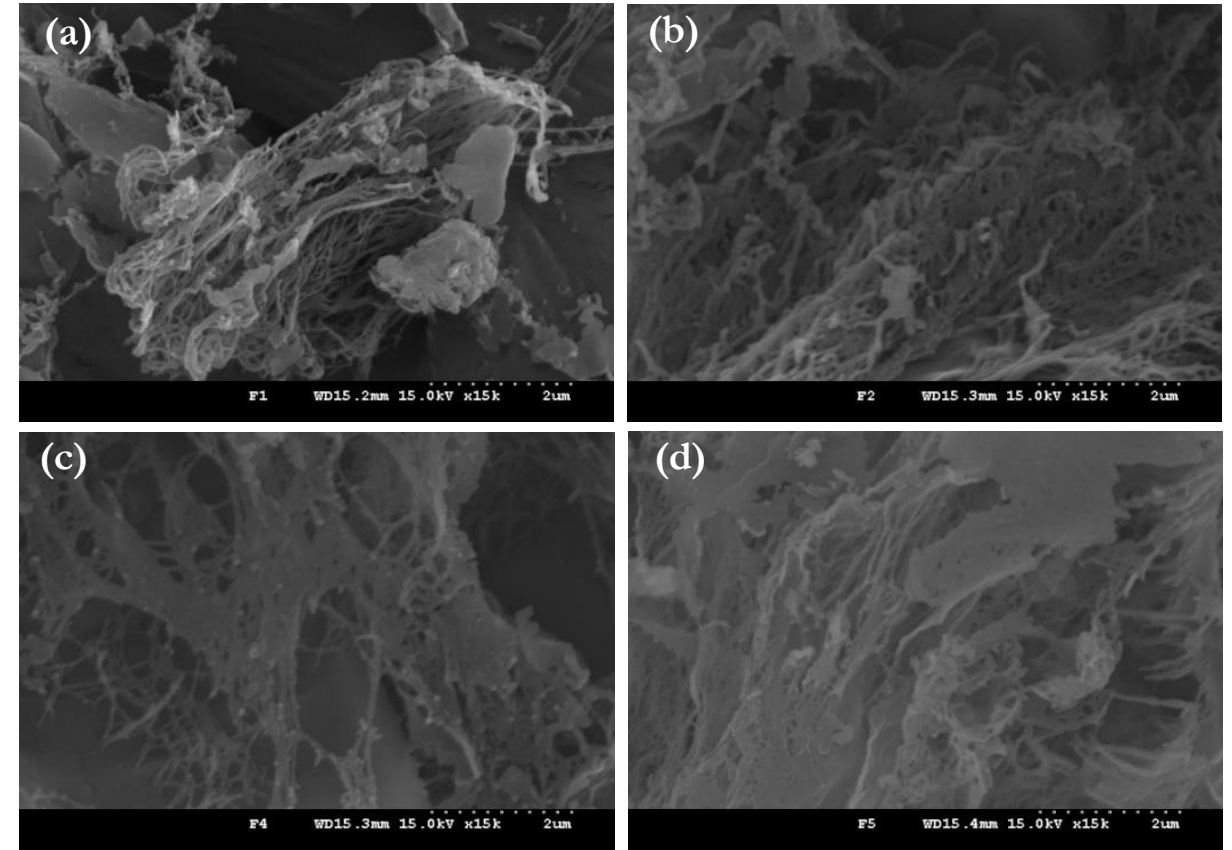

Figure 2: FESEM images of CS-NFS/HMW at different molar ratios (a) 10, (b) 20, (c) 30 and (d) 40

\subsection{FTIR Spectroscopy}

The FTIR spectra of CS-NFS were displayed in Fig.3 (a-d) at different concentrations from 10, 20, 30 and $40 \mathrm{mM}$ (chitosan monomer). The characteristic bands $\sim 3200$ and $3420 \mathrm{~cm}^{-1}$ due to the stretching vibration of $\mathrm{OH}$ and $\mathrm{NH}_{2}$ groups of chitosan and the bands at 2900 and $1540 \mathrm{~cm}^{-1}$ represented the presence of $-\mathrm{CH}$ and $-\mathrm{CH}_{2}$ group, $1444 \mathrm{~cm}^{-1}$ peak is due to $\mathrm{C}-\mathrm{N}$ axial deformation (amine group band). The typical band around $1085 \mathrm{~cm}^{-1}$ peak represents the $\mathrm{C}-\mathrm{O}$ groups of chitosan. The peak at $1570 \mathrm{~cm}^{-1}$ is attributed to the N$\mathrm{H}$ deformation of $\mathrm{NH}_{2}$ group and $1029 \mathrm{~cm}^{-1}$ stretching vibration of $\mathrm{C}-\mathrm{O}-\mathrm{C}$ in glucose circle and 1062-1010 $\mathrm{cm}^{-1}$ band corresponds to $\mathrm{CH}-\mathrm{OH}$ in cyclic compounds [30]. Hence, these spectral evidences further augment the existence of chitosan units in the nanofibrous scaffolds.

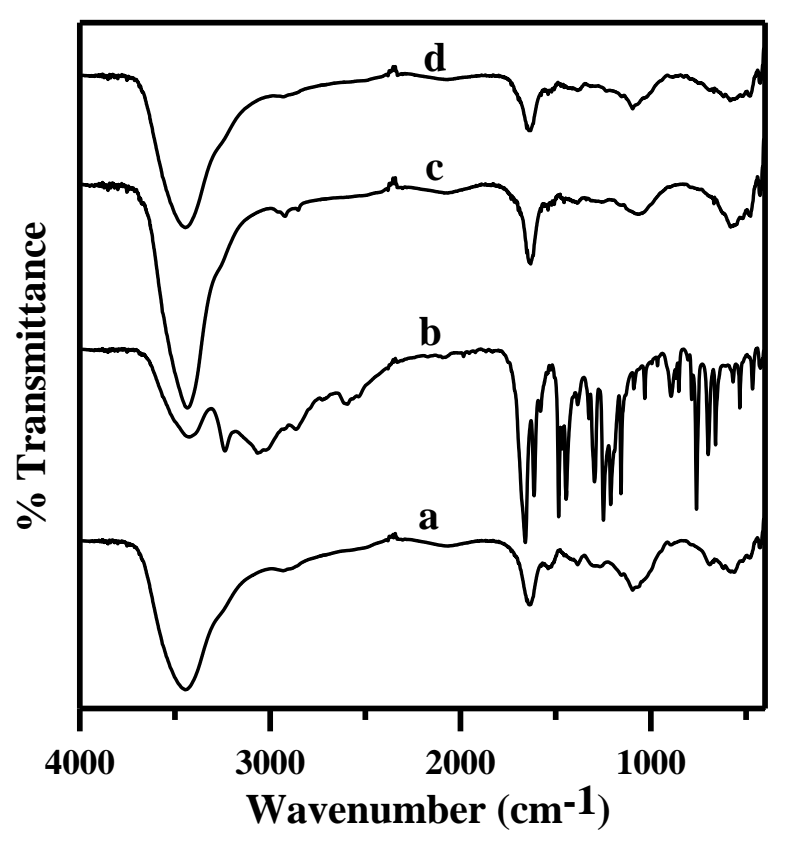

Figure 3: FTIR spectra of CS-NFS/LMW at different molar ratios (a) 10, (b) 20, (c) 30 and (d) 40 


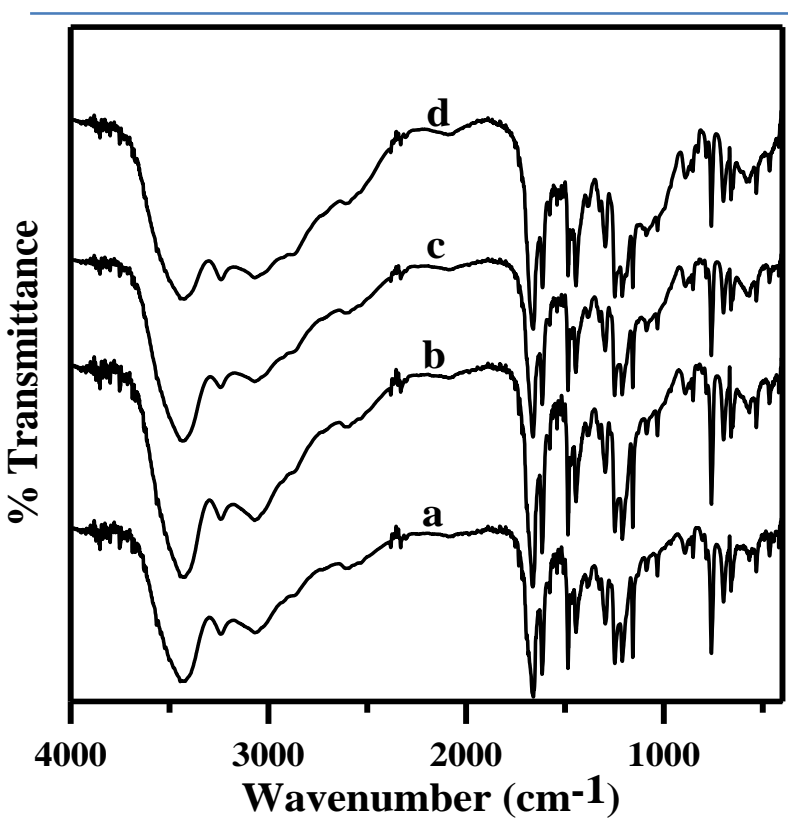

Figure 4: FTIR spectra of CS-NFS/HMW at different molar ratios (a) 10, (b) 20, (c) 30 and (d) 40

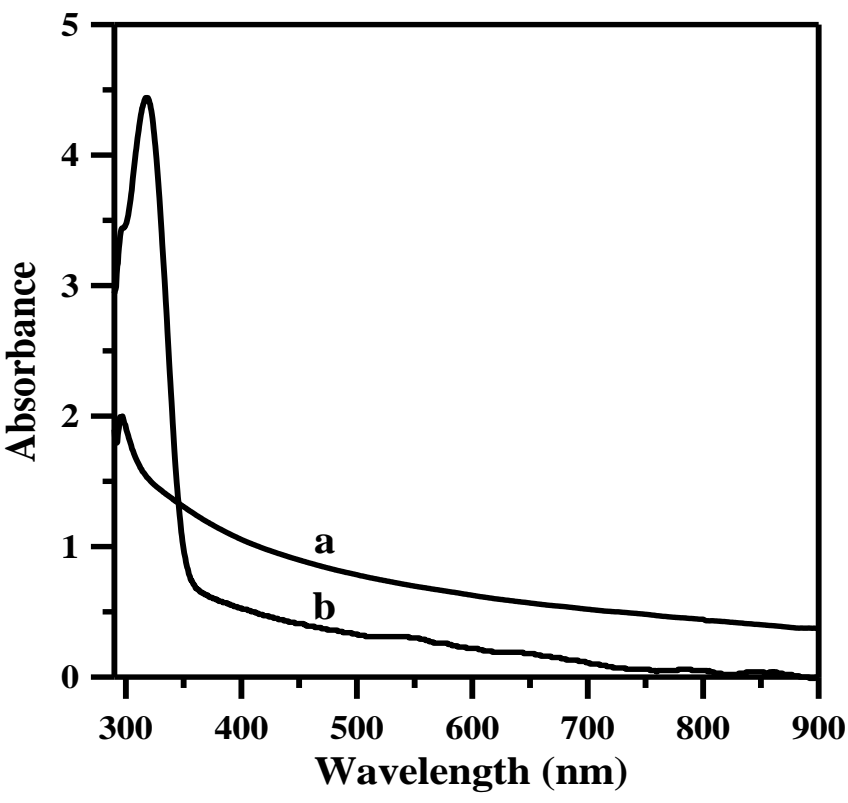

Figure 5: $U V$-visible spectra of (a) CS-NFS/LMW and (b) CS-NFS/HMW

Fig.4. displays the FTIR spectra of the CS-NFS/HMW. Through the exploit of a combination of the OH stretching band at $3440 \mathrm{~cm}^{-1}$, amide I bands at 1654 and $1625 \mathrm{~cm}^{-1}$, amide II band at $1570 \mathrm{~cm}^{-1}, \mathrm{C}-\mathrm{H}$ stretching band at $2871 \mathrm{~cm}^{-1}$, the bridge oxygen stretching band at $1156 \mathrm{~cm}^{-1}$, and the C-O stretching band at 1065 and $1032 \mathrm{~cm}^{-1}$, the percent deacetylation can be determined [31].

\subsection{UV-Visble Spectroscopy}

Fig. 5 presents the UV-visible spectrum of CS-NFS at low and high molecular weight. The absorption band seen at $\sim 300$ (Fig.5a) to $320 \mathrm{~nm}$ (Fig.5b) arising due to the $\pi-\pi^{*}$ transition of $-\mathrm{C}=\mathrm{O}$ group of chitosan degradation [32]. Hence, this evidence confirms the existences of chitosan units in the nanofibrous matrix.

\subsection{Thermal Studies}

Thermal stability of the CS-NFS were evaluated with TG analysis (Fig.6 and 7 (a-d). TG analysis was carried out for all the NFM s from the temperature 25$800^{\circ} \mathrm{C}$, ca. TG analysis of the CS-NFS confirms the presence of both polymer fractions in the fibers. The primary weight loss $100^{\circ} \mathrm{C}$ is due to loss of solvent and water from the chitosan nanofibers [3335]. The second thermal degradation at $240^{\circ} \mathrm{C}$ is owing to the decomposition of chitosan nanofibers. Thus, this thermal evidence further confirms the chitosan units in the fibrous matrix.

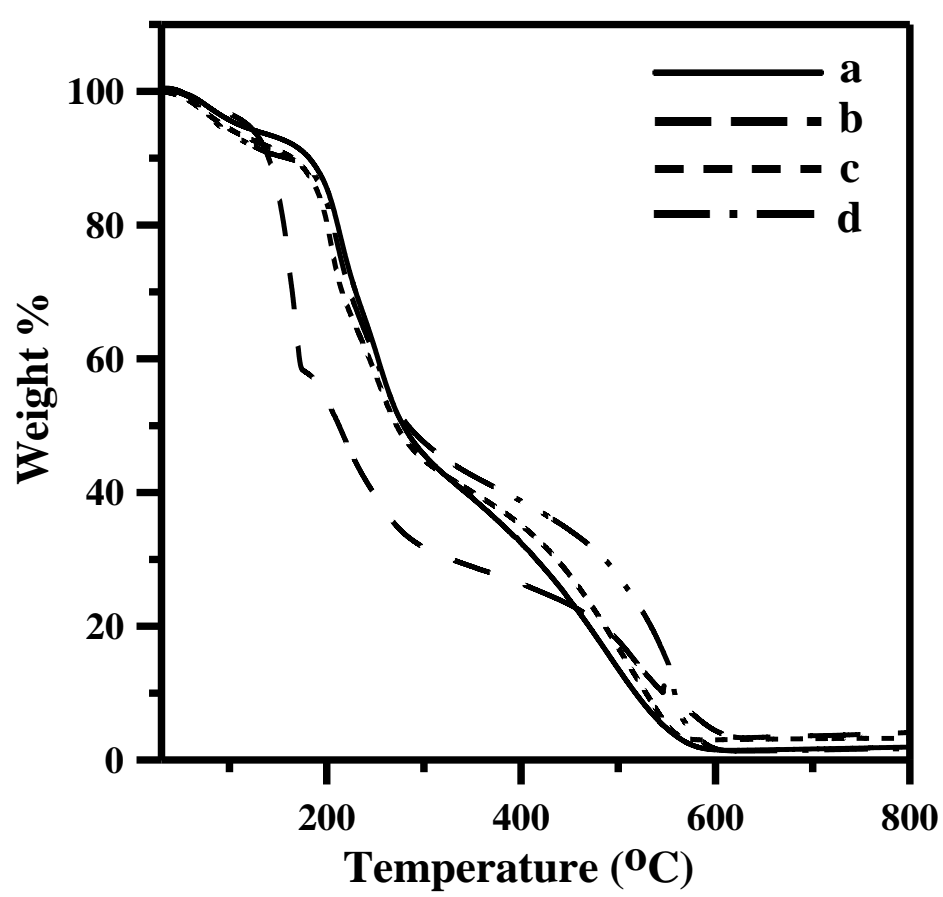

Figure 6: TGA curves of CS-NFS/LMW at different molar ratios (a) 10, (b) 20, (c) 30 and (d) 40 
Figure 7:

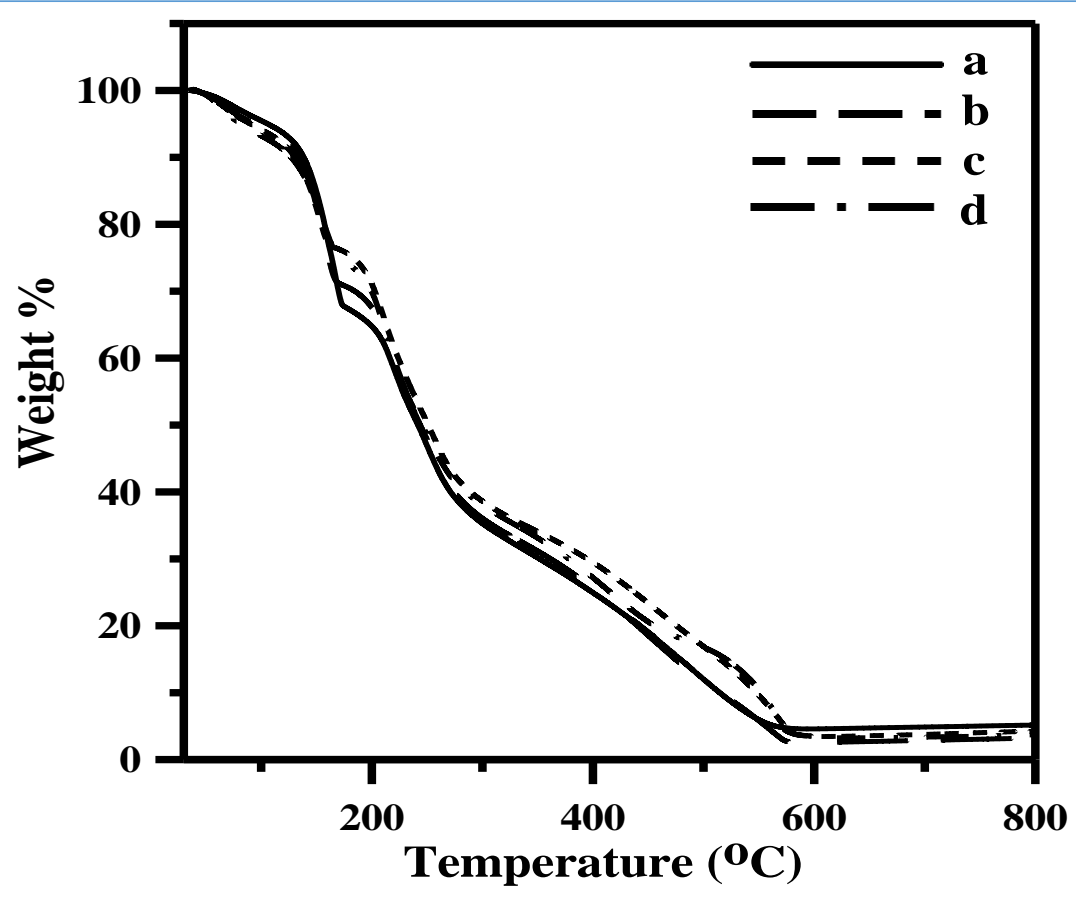

CS-NFS/HMW at different molar ratios (a) 10, (b) 20, (c) 30 and (d) 40

TGA curves of

\section{Conclusions}

It was able to obtain bead-free, continuous, randomly oriented nanofibrous matrix through Oil/Water Emulsion method from various CS molecular weights (low and high). The fiber diameter and molar ratio of CS are correlated. The morphology of the nanofibrous matrix was examined using field emission scanning electron microscopy. The diameter of the fiber is in the range of $\sim 100-200 \mathrm{~nm}$. The structural features were characterized by UV-visible and FTIR spectroscopy. The thermal properties of the fibers also discussed. Our technology for effective production of the chitosan nanofibrous matrix will contribute to the advanced functional materials and anticipated to be useful for biosensors, tissue engineering and membrane science.

\section{Acknowledgement}

The authors gratefully acknowledge the support from Science and Engineering Research Board Department of Science Technology grant (SB/FT/CS-117/2014). The authors acknowledge the basic research support from National Institute of Technology Puducherry, Karaikal, India.

\section{How to Cite this Article:}

R. Dhanusuraman, E. Muthusankar, and D. Kamalakannan, "Facile Synthesis and Characterization of Chitosan Nanofibers by Oil/Water Emulsion Method”, Advanced Nano Research, vol. 1, no. 1, pp. 31-37, Apr. 2018. doi: 10.21467/anr.1.1.31-37

\section{References}

[1] Zhang, Lijie, and Thomas J. Webster, "Nanotechnology and nanomaterials: promises for improved tissue regeneration", Nano today, 4(1), 66-80, 2009.

[2] Ohkawa, Kousaku, Ken-Ichi Minato, Go Kumagai, Shinya Hayashi, and Hiroyuki Yamamoto, "Chitosan nanofiber", Biomacromolecules, 7(11), 3291-3294,2006.

[3] X. Geng, O.H. Kwon and J. Jang, "Electrospinning of chitosan dissolved in concentrated acetic acid solution", Biomaterials, 26,5427, 2005.

[4] Wang, Jian-Wen, Ching-Yi Chen, and Yi-Ming Kuo, "Chitosan-poly (acrylic acid) nanofiber networks prepared by the doping induction of succinic acid and its ammonia-response studies", Polymers for Advanced Technologies, 19(9), 1343-1352, 2008.

[5] Marsich, Eleonora, Massimiliano Borgogna, Ivan Donati, Pamela Mozetic, Berit L. Strand, Santiago Gomez Salvador, Franco Vittur, and Sergio Paoletti, "Alginate/lactose-modified chitosan hydrogels: A bioactive biomaterial for chondrocyte encapsulation", Journal of biomedical materials research, Part A 84(2), 364-376, 2008. 
Dhanusuraman et al., Adv. Nan. Res.; Vol. 1, Issue 1, pp: 31-37, 2018

[6] N, Canas, T. Valero, M. Villarroya, E. Montell, J. Verges, A.G. Garcia and M.G. Lopez, "Chondroitin sulfate protects SH-SY5Y cells from oxidative stress by inducing heme oxygenase-1 via phosphatidylinositol 3-kinase/Akt", Journal of Pharmacology and Experimental Therapeutics,323, 946, 2007.

[7] Lahiji, Ashkan, Afshin Sohrabi, David S. Hungerford, and Carmelita G. Frondoza, "Chitosan supports the expression of extracellular matrix proteins in human osteoblasts and chondrocytes", Journal of biomedical materials research, 51(4), 586-595, 2000.

[8] Shahidi, Fereidoon, Janak Kamil Vidana Arachchi, and You-Jin Jeon, "Food applications of chitin and chitosans", Trends in food science \& technology, 10(2), 37-51, 1999.

[9] He, Ping, Stanley S. Davis, and Lisbeth Illum, "In vitro evaluation of the mucoadhesive properties of chitosan microspheres", International journal of pharmaceutics, 166(1), 75-88,1998.

[10] Ilyina, A. V., V. E. Tikhonov, A. I. Albulov, and V. P. Varlamov, "Enzymic preparation of acid-free-water-soluble chitosan", Process Biochemistry, 35(6),563-568, 2000.

[11] Jumaa, Muhannad, and Bernd W. Müller, "Physicochemical properties of chitosan-lipid emulsions and their stability during the autoclaving process", International journal of pharmaceutics, 183(2), 175-184,1999.

[12] Du, Wen-Li, Shan-Shan Niu, Ying-Lei Xu, Zi-Rong Xu, and Cheng-Li Fan, "Antibacterial activity of chitosan tripolyphosphate nanoparticles loaded with various metal ions", Carbohydrate Polymers, 75(3),385-389,2009.

[13] Wan, Ying, Xiaoying Cao, Quan Wu, Shengmin Zhang, and Sheng Wang, "Preparation and mechanical properties of poly (chitosang-DL-lactic acid) fibrous mesh scaffolds", Polymers for Advanced Technologies, 19(2), 114-123,2008.

[14] Sogias, Ioannis A., Adrian C. Williams, and Vitaliy V. Khutoryanskiy, "Why is chitosan mucoadhesive?", Biomacromolecules, 9(7),1837-1842,2008.

[15] Khor, Eugene, "Chitin: a biomaterial in waiting”, Current Opinion in Solid State and Materials Science, 6(4),313-317,2002.

[16] Rinaudo, Marguerite, "Main properties and current applications of some polysaccharides as biomaterials", Polymer International, 57(3), 397-430,2008.

[17] Yang, Anle, and Renjie Wu, "Mechanical properties and interfacial interaction of a novel bioabsorbable chitin fiber reinforced poly ( $\in$-caprolactone) composite”, Journal of materials science letters, 20(11)977-979,2001.

[18] S.C. Anand, J.F. Kennedy, M. Miraftab and S. Rajendran, Medical Textiles and Biomaterials for Healthcare, Woodhead Publishing Ltd, (2006)

[19] Qin, Yimin, "The preparation and characterization of chitosan wound dressings with different degrees of acetylation", Journal of applied polymer science, 107(2),993-999,2008.

[20] Burkatovskaya, Marina, George P. Tegos, Emilia Swietlik, Tatiana N. Demidova, Ana P. Castano, and Michael R. Hamblin, "Use of chitosan bandage to prevent fatal infections developing from highly contaminated wounds in mice", Biomaterials, 27(22), 41574164, 2006.

[21] Lim, Sang-Hoon, and Samuel M. Hudson, "Application of a fiber-reactive chitosan derivative to cotton fabric as an antimicrobial textile finish", Carbohydrate Polymers, 56(2), 227-234, 2004.

[22] Liu, Guojun, Jianfu Ding, Lijie Qiao, Andrew Guo, Boris P. Dymov, James T. Gleeson, T. Hashimoto, and K. Saijo, "Polystyreneblock-poly (2-cinnamoylethyl methacrylate) Nanofibers-Preparation, Characterization, and Liquid Crystalline Properties", Chemistry-A European Journal, 5(9),2740-2749,1999.

[23] McCulloch, W. J. G, "The history of the development of melt blowing technology", International Nonwovens Journal, 8(1),6672,1999 .

[24] Ondarcuhu, T., and C. Joachim, "Drawing a single nanofibre over hundreds of microns", EPL (Europhysics Letters), 42(2),215,1998.

[25] Feng, Lin, Shuhong Li, Huanjun Li, Jin Zhai, Yanlin Song, Lei Jiang, and Daoben Zhu, "Super-hydrophobic surface of aligned polyacrylonitrile nanofibers", Angewandte Chemie, 114(7), 1269-1271,2002.

[26] Blair, Richard, Heather Shepherd, Tanya Faltens, Philip C. Haussmann, Richard B. Kaner, Sarah H. Tolbert, Jiaxing Huang, Shabnam Virji, and Bruce H. Weiller, "Construction of a polyaniline nanofiber gas sensor", Journal of Chemical Education, 85(8),1102, 2008.

[27] Zhang, Xinyu, Roch Chan-Yu-King, Anil Jose, and Sanjeev K. Manohar, "Nanofibers of polyaniline synthesized by interfacial polymerization", Synthetic Metals, 145(1), 23-29, 2004.

[28] Du, Jimin, Jianling Zhang, Buxing Han, Zhimin Liu, and Meixiang Wan, "Polyaniline microtubes synthesized via supercritical CO2 and aqueous interfacial polymerization", Synthetic metals, 155(3),523-526,2005.

[29] Liu, Siwei, Kaizheng Zhu, Yi Zhang, and Jiarui Xu, "Cyclic polyaniline nanostructures from aqueous/organic interfacial polymerization induced by polyacrylic acid", Polymer, 47(22), 7680-7683,2006.

[30] Sangsanoh, Pakakrong, and Pitt Supaphol, "Stability improvement of electrospun chitosan nanofibrous membranes in neutral or weak basic aqueous solutions", Biomacromolecules, 7(10), 2710-2714, 2006

[31] Shigemasa, Yoshihiro, Hiroaki Matsuura, Hitoshi Sashiwa, and Hiroyuki Saimoto, Evaluation of different absorbance ratios from infrared spectroscopy for analyzing the degree of deacetylation in chitin", International Journal of Biological Macromolecules, 18(3) 237-242, 1996.

[32] Kaushik, Ajeet, Raju Khan, Pratima R. Solanki, Pratibha Pandey, Javed Alam, Sharif Ahmad, and B. D. Malhotra, "Iron oxide nanoparticles-chitosan composite based glucose biosensor", Biosensors and bioelectronics, 24(4), 676-683, 2008.

[33] Yue, J., A. J. Epstein, Z. Zhong, P. K. Gallagher, and A. G. MacDiarmid, "Thermal stabilities of polyanilines", Synthetic Metals, 41(1-2),765-768,1991.

[34] Ragupathy, Dhanusuraman, Palanisamy Gomathi, Loganathan Kumaresan, Soo Chool Lee, Salem S. Al-Deyab, Sang Hak Lee, and Han Do Ghim, "Self-assembly growth of electrically conductive chitosan nanofibrous scaffold", Macromolecular Research, 20(10),1070-1074,2012.

[35] Gomathi, Palanisamy, Min Kwan Kim, Jung Je Park, Dhanusuraman Ragupathy, Annamalai Rajendran, Soo Chool Lee, Jae Chang Kim, Sang Hak Lee, and Han Do Ghim, "Multiwalled carbon nanotubes grafted chitosan nanobiocomposite: A prosperous functional nanomaterials for glucose biosensor application", Sensors and Actuators B: Chemical, 155(2),897-902,2011. 